\title{
Tracheal granuloma after thyroidectomy with difficult intubation
}

\author{
Eun-Jeong Cho, Chang Jae Kim, Myung No Lee, and Mee Young Chung \\ Department of Anesthesiology and Pain Medicine, College of Medicine, The Catholic University of Korea, Seoul, Korea
}

Tracheal complications after endotracheal intubation or tracheostomy include tracheal stenosis, ulcers, granulomas and tracheomalacia [1]. Of these, tracheal granuloma is a rare complication, and common on the anterior wall of the subglottis [2]. We experienced a case in which a patient with symptoms of airway obstruction due to tracheal granuloma after total thyroidectomy underwent resection of a tracheal granuloma and anastomosis under general anesthesia after bronchoscopy.

A 55-year-old male patient had undergone total thyroidectomy due to thyroid cancer. However, symptoms of upper respiratory infection, cough and sputum, continued after discharge. After 4 months, stridor and dyspnea developed, and flexible bronchoscopy was performed to assess. A granuloma about $1 \mathrm{~cm} \times 1 \mathrm{~cm}$ in size was found $3 \mathrm{~cm}$ below the vocal cords on fiberoptic bronchoscopy. The patient was transferred to our hospital for diagnosis and treatment using interventional rigid bronchoscopy. According to the history of the first operation, the patient was $166 \mathrm{~cm}$ tall and, weighed $73 \mathrm{~kg}$, with a body mass index of 26 , classified as, mildly overweight. He had had predictive signs of difficult intubation: short neck, Mallampati class III, thyromental distance of 2 fingerbreadths. He had not had any respiratory symptoms. A $1.5 \mathrm{~cm} \times 1.0 \mathrm{~cm}$-sized thyroid cancer was founded, but no abnormal findings at any other sites were noted on neck computed tomography (CT) scan. On laryngoscopy, the patient's airway was classified as CormackLehane grade IIIa. His trachea was intubated using an ID 6.5 $\mathrm{mm}$ endotracheal tube with stylet after trials with an ID 8.0 $\mathrm{mm}$ reinforced an endotracheal tube and an ID $7.0 \mathrm{~mm}$ plain endotracheal tube with inserted stylet. The cuff volume was about $5 \mathrm{cc}$. Anesthesia was adequately maintained with $\mathrm{N}_{2} \mathrm{O}-\mathrm{O}_{2}-$ sevoflurane at bispectral index 44-58 during the thyroidectomy. The surgeon placed a $10 \mathrm{~cm}$ high pillow under the shoulder and extended the neck simultaneously for the thyroid surgery. The operating time for the thyroidectomy was 84 minutes.

In our hospital, CT scan revealed a polypoid mass about $1 \mathrm{~cm}$ in length with erosion of cartilage in the right lateral aspect of the upper trachea, along with tracheal stenosis (Fig. 1A). To facilitate the performance of rigid bronchoscopy, propofol and remifentanil were used with rocuronium as anesthetic agents. However, the rigid bronchoscopy could not be performed because the patient had a short neck and limited neck extension. Therefore, the tracheal granuloma was inspected by flexible bronchoscopy instead of rigid bronchoscopy (Fig. 1B), and the trachea was intubated by guided flexible bronchoscopy using an ID $6.5 \mathrm{~mm}$ endotracheal tube. The decision was made to perform surgery on the patient in the Department of Thoracic Surgery on the following day.

During the second operation, the tracheal region including the granuloma was resected and the trachea was anastomosed under general anesthesia using $\mathrm{N}_{2} \mathrm{O}-\mathrm{O}_{2}$-sevoflurane. A $1.0 \mathrm{~cm}$ $\times 1.0 \mathrm{~cm}$-sized granuloma was located on the lower cricoid cartilage and stenotic change was observed around the granuloma. As a result of this tissue biopsy, the mass was diagnosed as a typical granuloma with ulcer and sclerosis.

Because this patient had undergone thyroidectomy due to thyroid cancer, we assumed that a mass in the trachea might be caused by extended cancer. However, the mass was located at the region of the tube-tip and had a yellowish and smooth sur-

Corresponding author: Mee Young Chung, M.D., Department of Anesthesiology and Pain Medicine, College of Medicine, The Catholic University of Korea, 620-56, Jeonnong-2dong, Dongdaemun-gu, Seoul 130-709, Korea. Tel: 82-2-958-2099, Fax: 82-2-967-0235, E-mail: jhjs0806@catholic.ac.kr (c) This is an open-access article distributed under the terms of the Creative Commons Attribution Non-Commercial License (http:// creativecommons.org/licenses/by-nc/3.0/), which permits unrestricted non-commercial use, distribution, and reproduction in any medium, provided the original work is properly cited. 

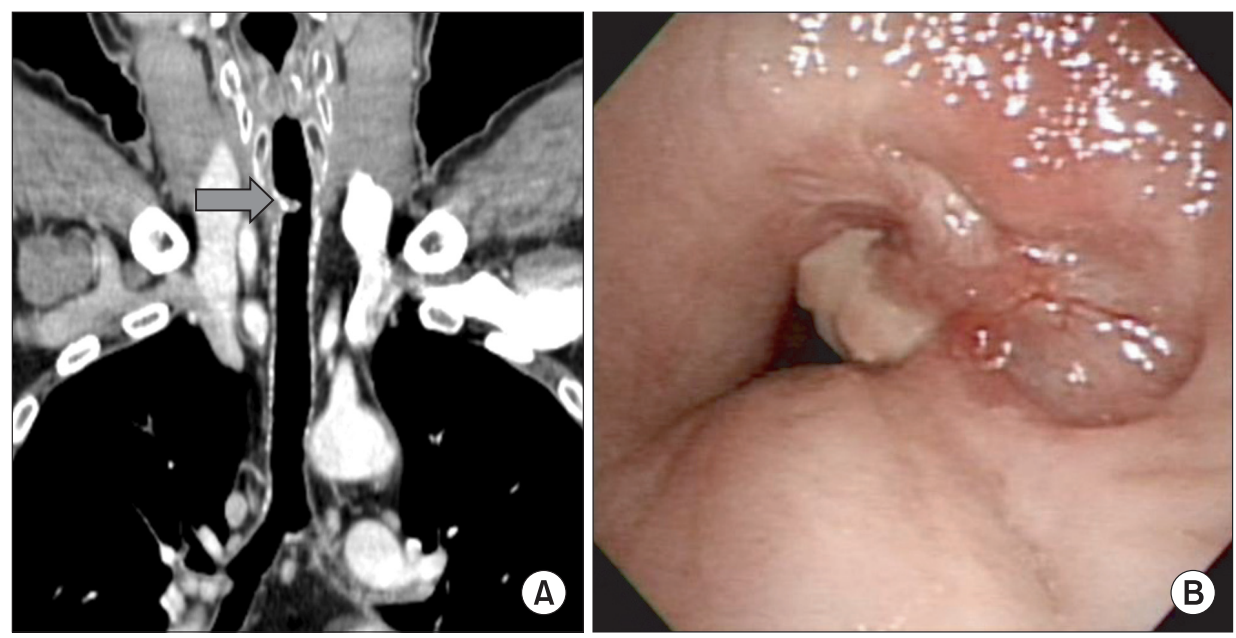

Fig. 1. (A) A polypoid mass (arrow) is shown in the right lateral aspect of the upper trachea on computed tomography scan. (B) Tracheal granuloma with stenotic change is noted about $3 \mathrm{~cm}$ below the vocal cords on fiberoptic bronchoscopy.

face. We could consider this benign mass, a granuloma, which was caused by traumatic intubation. Tracheal granulomas can form from irritation caused by contact with an endotracheal tube which is placed between the vocal folds and the trachea. Etiologic factors that contribute to tracheal obstructive lesions include tracheal ischemia due to high cuff pressure, traumatic intubation, the use of an oversized tube, duration of intubation, positioning of the endotracheal tube, and cardiovascular status during intubation $[3,4]$. Tracheal mucosal damage in this patient might have been caused by traumatic intubation because intubation was attempted three times and the stylet was used to allow direction through an unseen glottis. In addition, tube-tip movement due to mechanical ventilation under the position of the thyroid and the manipulation of the thyroid during surgery might also have lead to tracheal damage in this case. Other etiologic factors, such as cuff pressure, size of the tube and duration of intubation, and cardiovascular status, might not have played a role in this patient's tracheal damage. Predisposing factors include female gender, obesity, short neck, congenital anomalies involving the airway, fragility of the laryngotracheal mucosa, and hyperacidity with gastric reflux [3]. Our patient's predisposing factors were obesity and short neck.

Symptoms of dry cough and difficulty in raising sputum may indicate a moderate tracheal stenosis or lesion. Dyspnea and stridor are caused when more than $75 \%$ of the tracheal lumen is stenosed or when the diameter of the tracheal lumen is reduced to $5 \mathrm{~mm}$ [4]. The patient in this case had dyspnea and stridor when about $70 \%$ of the tracheal lumen was stenosed; the diameters of the mass and trachea were about $1 \mathrm{~cm}$ and $1.4 \mathrm{~cm}$, respectively. Therefore, we guessed that his respiratory difficulty was worsened because the granuloma was soft and might move along with breathing. Diagnostic methods include spirometry, CT scan, and bronchoscopy. A simple bronchoscopic procedure is used to classify tracheo-broncheal stenosis and to compare the results of a wide range of interventions with the outcomes [5].

Although a steroid regimen can successfully treat a patient with tracheal stenosis, tracheal reconstruction is the main treatment for symptomatic lesions. However, the mortality rate for major surgery is $3 \%$ [5]. Recently, rigid bronchoscopy and tracheal dilatation, possibly with placement of a stent, have been proven effective in the treatment of less serious lesions, and may also provide the time to prepare for definitive procedures in more severe cases [5]. Patients with predisposing factors for laryngotracheal injury, such as difficult intubation or excessive flexion or extension of neck, should require routine tracheal assessment 4-6 weeks after extubation. In addition, in cases in which, severe tracheal stenosis may occur early, an endoscopic examination must be performed immediately in patients with even mild airway obstruction symptoms [4].

In summary, we experienced a case in which tracheal granuloma occurred after thyroidectomy with difficult intubation, was assessed by fiberoptic bronchoscopy and was resected successfully. We recommend that particular care be taken with intubation in patients with predisposing factors for laryngotracheal injury, and when any symptoms of airway obstruction occur, airway assessment with bronchoscopy is required. 


\section{References}

1. Kang HH, Kim JW, Kang JY, Kim JS, Kim MS, Kim SS, et al. Obstructive fibrinous tracheal pseudomembrane after tracheal intubation: a case report. J Korean Med Sci 2010; 25: 1384-6.

2. Harley HR. Laryngotracheal obstruction complicating tracheostomy and endotracheal intubation. A critical review. Thorax 1971; 26: 493533.

3. Kaneda M, Goto R, Ishijima S, Kawakami S, Park K, Shima Y. Laryngeal granuloma caused by short-term endotracheal intubation. Anesthesiology 1999; 90: 1482-3.

4. Kastanos N, Estopá Miró R, Marín Perez A, Xaubet Mir A, Agustí-Vidal A. Laryngotracheal injury due to endotracheal intubation: incidence, evolution, and predisposing factors. A prospective long-term study. Crit Care Med 1983; 11: 362-7.

5. Spittle N, McCluskey A. Lesson of the week: tracheal stenosis after intubation. BMJ 2000; 321: 1000-2. 